\title{
Role of Radiology in Central Hemangioma of jaws
}

\author{
Sanjeev Kumar Jindal ${ }^{1}$, Soheyl Sheikh ${ }^{2}$, Anshu Singla ${ }^{3}$, Nidhi Puri ${ }^{4}$
}

${ }^{1}$ Senior Lecturer, Department of Oral Medicine \& Radiology, Bhojia Dental College \& Hospital, Baddi, District Solan, Himachal Pradesh.

${ }^{2}$ Professor, Department of Oral Medicine and Radiology, M.M. College of Dental Sciences \& Research, Mullana, Ambala, India. ${ }^{3}$ Senior Lecturer, Department of Pedodontics \& Preventive Dentistry, Bhojia Dental College \& Hospital, Baddi, District Solan, Himachal Pradesh.

${ }^{4}$ B.D.S., P.G. Student, Department of Oral Medicine and Radiology, M.M. College of Dental Sciences \& Research, Mullana, Ambala, India.

Jindal SK, Sheikh S, Singla A, Puri N. Role of Radiology in Central Hemangioma of jaws. J Clin Exp Dent. 2010;2(2):e76-8.

http://www.medicinaoral.com/odo/volumenes/v2i2/jcedv2i2p76.pdf

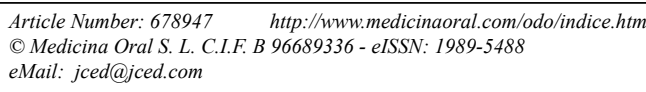

\begin{abstract}
Central hemangioma, a bone destructive lesion, is a benign tumor. Hemangiomas of jaws are rare entity and produces many different radiographic images. Radiographic differential diagnosis includes osteosarcoma, fibrous dysplasia, central giant cell granuloma, ameloblastoma, odontogenic myxoma, multiple myeloma, dentigerous cyst and aneurysmal bone cyst. Since it involves proliferation of blood vessels so aspiration or biopsy of such lesions can lead to severe hemorrhage which can turn out to be lethal. We stress on the importance of radiology in the timely diagnosis of such lesions which can prevent the disaster not only to the patient but also to oral surgeon in legal and professional aspects.
\end{abstract}

Key words: Central hemangioma, jaws, radiography. 


\section{Introduction}

A hemangioma is a proliferation of blood vessels that creates a mass resembling a neoplasm. The central (intraosseous) type is frequently found in the vertebrae and skull and rarely develops in the jaws. The lesion produces a hard non tender slow-growing swelling (1). The teeth in the vicinity of the tumor may be loosened and bleeding may occur from the gingiva around the necks of the affected teeth. The teeth have increased hypermobility and may exhibit a pumping action such that, when depressed in an apical direction, the teeth rapidly resume their original position. The lesion may pulsate, and a bruit may be detected on auscultation. Some hemangiomas may be present without any sign or symptom (2). These lesions of bone have been referred to as "the great mimicker" because they can produce many different radiographic images. Most commonly, lesions show a multilocular radiolucency with small (honeycomb appearance) or large (soap-bubble appearance) loculations. Phleboliths appearing as small rounded or sausage shaped radiopacities having concentric structure with small radiolucent dot in the centre may be seen. Lesions may also present as a unilocular radiolucency (3).

Biopsy or surgical excision of lesion can result in severe hemorrhage which poses a risk of lethal exsanguinations of the patient to the point of death; therefore a correct clinical diagnosis is desirable before any surgical intervention. Therefore, dental practitioners should be aware of the radiological features of this vascular bone lesion. The purpose of this paper is to critically evaluate the radiological and clinical features of central hemangioma of the jaws from the literature.

\section{Epidemiology and Pathogenesis}

Hemangiomas of the jaws are quite rare; when they occur two thirds are found in the mandible, with a female: male ratio of 2:1 and peak incidence in the second decade of life $(4,5)$. In the mandible, the greatest frequency of occurrence has been the body region but condylar tumors have also been reported (1). The origin of central hemangioma is debatable. Some believe that it is a true benign neoplasm, while others state that it is a hamartoma resulting from proliferation of mesoderm cells that undergo endothelial differentiation and subsequently are canalized and vascularized $(4,5)$. Malignant transformation of central hemangioma has been reported in some cases. Most central haemangiomas are the cavernous type (large-calibre vessels), but can be the capillary type (small-calibre vessels) (5).

\section{Clinical Picture}

Signs and symptoms include discomfort, oozing or pulsatile bleeding from the gingiva around the teeth in the region of the lesion, bluish discoloration of gingiva, mobile teeth, derangement of arch form, accelera- ted exfoliation and agenesis of teeth. Highly expansile lesions cause sensation of pulsation, audible bruits on extension into the soft tissue and blanching on pressure. Occasionally patients have paraesthesia in the region of the lesion $(5,6)$.

\section{Differential Diagnosis}

Radiographic differential diagnosis includes osteosarcoma, fibrous dysplasia, central giant cell granuloma, ameloblastoma, odontogenic myxoma, multiple myeloma, dentigerous cyst and aneurysmal bone cyst.

Osteosarcoma produces similar sunburst appearance. It may be entirely radiolucent, mixed radiolucent-radiopaque or quite radiopaque. There may be asymmetric broadening of periodontal ligament space and onion skin growth of periosteal bone. Irregular margins of a lesion raise a question of malignant tumour like osteosarcoma (5).

Central haemangioma with areas of increased radiopacity can be mistaken for ground glass appearance of fibrous dysplasia. The other radiolucent patterns of central haemangioma can be misdiagnosed as intermediate stages of fibrous dysplasia but this lesion does not show sunburst appearance.

Multilocular regions of rarefaction that accompany expansion and thinning of the cortex may mimic a central giant cell granuloma of the jaws. However some investigators believe that loculations produced by a central haemangioma are smaller with fine fibrillar network.

Cystic lesions are more difficult to diagnose radiographically and an aneurysmal bone cyst may be suspected, but radiographically this lesion does not resorb the adjacent teeth (7).

Since haemangioma of bone occurs early in life, the lesion can be in close proximity to the erupting teeth and it can be mistaken for a dentigerous cyst. In contrast to haemangioma, a dentigerous cyst will be associated with a well corticated pericoronal radiolucency attached to the cementoenamel junction of impacted tooth $(6,7)$.

The central haemangioma of bone may be clinically and radiographically indistinguishable from another vascular condition known as central arteriovenous fistula, shunt or aneurysm.

Angiography has proved to be useful as a diagnostic tool when clinical and radiographic characteristics suggest a diagnosis of hemangioma. This will demonstrate presence of a vascular lesion and delineate its boundaries.

\section{Management}

The choice of treatment depends on the size and location of the lesion, age of the patient and anticipated problems. The range of treatment includes steroid therapy, carbon dioxide and argon laser therapy, sclerosing agents, irradiation, surgical excision with and without ligation of vessels and embolisation. 
Such cases should be dealt with $100 \%$ care and caution. Every case should be thoroughly studied and investigated before carrying out the treatment plan. Ignoring even a tiny aspect at any step can bring disaster not only to patient but also to surgeon in legal and professional aspects.

\section{References}

1. Marwah N, Agnihotri A, Dutta S. Central hemangioma: an overview and case report. Pediatr Dent. 2006;28:460-6.

2. Whear NM. Condylar haemangioma--a case report and review of the literature. Br J Oral Maxillofac Surg. 1991;29:44-7.

3. Zlotogorski A, Buchner A, Kaffe I, Schwartz-Arad D. Radiological features of central haemangioma of the jaws. Dentomaxillofac Radiol. 2005;34:292-6.

4. Alves S, Junqueira JL, de Oliveira EM, Pieri SS, de Magalhães MH, Dos Santos Pinto D Jr, et al. Condylar hemangioma: report of a case and review of the literature. Oral Surg Oral Med Oral Pathol Oral Radiol Endod. 2006;102:e23-7.

5. Nagpal A, Suhas S, Ahsan A, Pai K, Rao N. Central haemangioma: variance in radiographic appearance. Dentomaxillofac Radiol. 2005;34:120-5.

6. Bunel K, Sindet-Pedersen S. Central hemangioma of the mandible. Oral Surg Oral Med Oral Pathol. 1993;75:565-70.

7. Beziat JL, Marcelino JP, Bascoulergue Y, Vitrey D. Central vascular malformation of the mandible: a case report. J Oral Maxillofac Surg. 1997;55:415-9. 